\title{
Good News für die Verhaltenstherapie
}

\section{Ulrich Voderholzer}

Schön Klinik Roseneck, Prien am Chiemsee, Deutschland
Sehr geehrte Kolleginnen und Kollegen,

mit diesem Editorial möchten wir Sie über gleich mehrere sehr erfreuliche Entwicklungen in Bezug auf die Zeitschrift VerhaltenSTHERAPIE informieren. Wie Ihnen sicher bekannt ist, spielt der sogenannte Impact Factor (IF) für die wissenschaftliche Bedeutung einer Zeitschrift eine besonders wichtige Rolle. Der IF zeigt an, in welchem Maße Veröffentlichungen aus einer Zeitschrift von anderen Wissenschaftlern in ihren Arbeiten innerhalb eines bestimmten Zeitraums berücksichtigt, d.h. zitiert werden. Hier haben es deutschsprachige Zeitschriften generell viel schwerer als englische Publikationsorgane, da sie weniger zitiert werden.

Umso mehr freuen wir uns, dass die letzte Analyse für 2018 (IF 2017) einen deutlich gestiegenen IF von 1,239 für die VERHALTENSTHERAPIE ergeben hat. Damit gehört unser Journal zu den deutschsprachigen wissenschaftlichen Fachzeitschriften im Bereich der psychischen Erkrankungen mit den höchsten IFs.

Dies bestätigt die Herausgeber darin, dass es gelungen ist, Autoren mit den richtigen Themen und Artikel von hoher Qualität für die Zeitschrift zu gewinnen. Aber wir glauben, da ist noch mehr möglich.

Eine weitere überaus erfreuliche Nachricht ist, dass sich Frau Prof. Dr. Christine Knaevelsrud, Berlin, und Frau Prof. Dr. Alexandra Philipsen, Bonn, als neue Herausgeberinnen der VerhaLTENSTHERAPIE angeschlossen haben.

Als sehr renommierte Wissenschaftlerinnen auf dem Gebiet der Verhaltenstherapie gehören sie seit 2018 dem Herausgebergremium an und werden dieses ab 2019 zunehmend aktiv verstärken.

Im Folgenden möchten wir die beiden neuen Herausgeberinnen kurz anhand der wichtigsten Stationen ihrer Lebensläufe und ihrer wissenschaftlichen Schwerpunkte vorstellen:

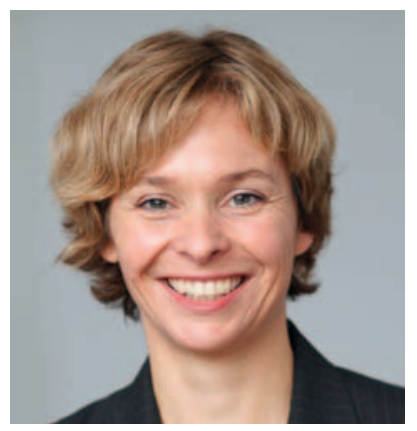

Prof. Dr. med. Alexandra Philipsen

Frau Prof. Alexandra Philipsen hat zunächst ihre Facharztweiterbildung und Psychotherapie-Ausbildung an der Klinik für Psychiatrie und Psychotherapie des Universitätsklinikums Freiburg absolviert, wo sie als Assistenzärztin, später als Oberärztin und in den Jahren 2011-2014 auch als geschäftsführende Oberärztin tätig war. Ihre klinischen und wissenschaftlichen Schwerpunkte stellten emotional instabile Persönlichkeitsstörungen und ganz besonders die Aufmerksamkeitsdefizit-/Hyperaktivitätsstörung (ADHS) im Erwachsenenalter dar, die auch das Thema ihrer Habilitation mit dem Titel «Ätiologie, Klinik und Therapie der Aufmerksamkeitsdefizit-/Hyperaktivitätsstörung im Erwachsenenalter» war. Weiterhin beschäftigte sich Frau Prof. Philipsen in ihrer Forschung schwerpunktmäßig mit der Neurobiologie der Emotionsregulation und Impulskontrolle sowie der Entwicklung und Evaluation störungsorientierter Psychotherapiekonzepte und pharmakologischer Studien zur Spannungsregulation und Impulskontrolle, insbesondere bei ADHS und der Borderline-Persönlichkeitsstörung. Von 2014-2017 war Frau Prof. Philipsen Ordinaria für Psychiatrie und Psychotherapie am Medizinischen Campus der Universität Oldenburg und Direktorin der Universitätsklinik für Psychiatrie und Psychotherapie an der Karl-Jaspers-Klinik in Bad Zwischenahn.

Seit 2018 ist Frau Prof. Philipsen Direktorin der Klinik für Psychiatrie und Psychotherapie am Universitätsklinikum Bonn und Lehrstuhlinhaberin für Psychiatrie und Psychotherapie an der Rheinischen Friedrich-Wilhelms-Universität Bonn.

\section{KARGER}

Fax +497614520714

\section{() 2018 S. Karger GmbH, Freiburg}




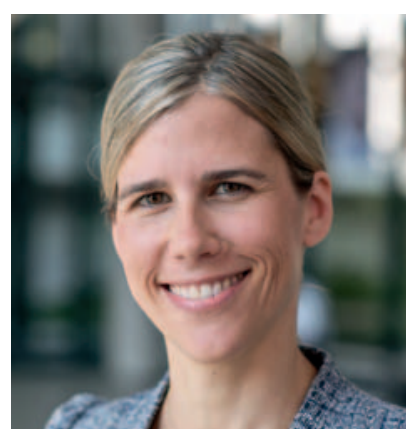

Prof. Dr. Christine Knaevelsrud

Frau Prof. Christine Knaevelsrud hat an der Universität Amsterdam und der New York University Psychologie studiert und anschließend an der Universität Zürich in der Arbeitsgruppe von Herrn Prof. Andreas Maercker promoviert.

Sie ist approbierte psychologische Psychotherapeutin und Professorin für Klinisch-Psychologische Intervention an der Freien Universität Berlin und hat von 2004-2010 eine Forschungsabteilung am Behandlungszentrum für Folteropfer geleitet. Frau Prof. Knaevelsrud hat mehrere multizentrische randomisiert-kontrollierte Interventionsstudien zur internetbasierten psychotherapeutischen Behandlung durchgeführt. Unter anderem wurden in diesen Studien die Störungsbilder «Depression», «Angststörungen», «posttraumatische Belastungsstörungen», «komplizierte Trauer» und «Anpassungsstörungen» untersucht. Die Populationen bestan- den aus Jugendlichen, älteren Menschen und arabischsprachigen Flüchtlingen. Für ihre Forschungsarbeit ist Frau Prof. Knaevelsrud mehrfach ausgezeichnet worden.

Eine der wichtigen Entwicklungen, auch in der Psychotherapie, ist die zunehmende Bedeutung von Internettherapien. Prof. Christine Knaevelsrud hat in Deutschland auf diesem Gebiet Pionierarbeit geleistet und mit Internettherapien für traumatisierte Flüchtlinge Angebote für Menschen entwickelt, die ansonsten gar keinen Zugang zur irgendeiner Form von Therapie gehabt hätten.

Mit Frau Prof. Knaevelsrud und Frau Prof. Philipsen wird unser Herausgeberteam weiter gestärkt - sowohl im Hinblick auf die Breite wissenschaftlicher Schwerpunkte, die in den letzten Jahren immer mehr an Bedeutung gewonnen hat, als auch bezüglich der Vernetzung der Zeitschrift in den Fachgebieten Psychiatrie und Psychotherapie sowie Psychologie und in der deutschen und internationalen Versorgungslandschaft.

Wir begrüßen die beiden neuen Herausgeberinnen herzlich und freuen uns auf die Zusammenarbeit.

Ulrich Voderholzer, Prien am Chiemsee, für die Herausgeber

\section{Disclosure Statement}

Es bestehen keine Interessenkonflikte. 